\title{
Primordial density perturbation in effective loop quantum cosmology
}

\author{
Golam Mortuza Hossain \\ The Institute of Mathematical Sciences, CIT Campus, Chennai-600113, India \\ and \\ Max-Planck-Institut für Gravitationsphysik, Albert-Einstein-Institut, Am Mühlenberg 1, \\ D-14476 Potsdam, Germany \\ E-mail: golam@imsc.res.in
}

Received 14 February 2005, in final form 4 May 2005

Published 6 June 2005

Online at stacks.iop.org/CQG/22/2511

\begin{abstract}
It is widely believed that a quantum field fluctuation in an inflating background creates the primeval seed perturbation which through subsequent evolution leads to the observed large-scale structure of the universe. The standard inflationary scenario produces a scale-invariant power spectrum quite generically but it produces, unless fine tuned, too large an amplitude for the primordial density perturbation observed. Using similar techniques, it is shown that a loop quantum cosmology induced inflationary scenario can produce a scale-invariant power spectrum as well as a small amplitude for the primordial density perturbation without fine tuning. Further, its power spectrum has a qualitatively distinct feature which is in principle falsifiable by observation and can distinguish it from the standard inflationary scenario.
\end{abstract}

PACS numbers: $04.60 . P p, 04.60 . \mathrm{Kz}, 98.80 . \mathrm{Jk}$

\section{Introduction}

The homogeneous and isotropic solution of the general theory of relativity, namely the Friedmann-Robertson-Walker (FRW) solution, appears to be an extremely good description of large-scale spacetime dynamics of our universe. The extreme simplicity of the FRW solution nevertheless ignores some crucial features such as it having certain sub-structure as well. On a large scale, the deviation from homogeneity and isotropy being small one can treat them as small perturbations around a homogeneous and isotropic background. The classical theory of large-scale structure formation can be used in principle to 'derive' the observed structure of the current universe but these models require us to know the initial seed perturbations. In this sense, the classical description of our universe is incomplete as there is no mechanism of generating the seed perturbation within the theory itself. 
On the other hand, the quantum field fluctuation in an inflating background quite generically produces a density perturbation with scale-invariant power spectrum [1] which is consistent with current observations. This is certainly an attractive feature of the standard inflationary scenario. However, one major problem that plagues almost all potential-driven inflationary scenarios is that these models generically produce too large an amplitude for the density perturbation, typically $\frac{\delta \rho}{\rho} \sim 1-10^{2}$ at horizon re-entry [2,3]. On the other hand, the cosmic microwave background (CMB) anisotropy measurements indicate $\frac{\delta \rho}{\rho} \sim 10^{-5}$. Naturally, to make these models viable it is necessary to fine tune the parameters of the field potential [4]. In the presence of quantum fluctuations it is rather difficult to justify or sustain this fine tuning of the field theoretical parameters.

It is worthwhile mentioning that inflation was invented to solve some crucial problems of the standard big bang (SBB) cosmology. The most important of them is the so-called particle horizon problem. The horizon problem is directly related to the fact that the standard model of cosmology contains an initial singularity where physical quantities such as energy density and spacetime curvature blow up leading to the breakdown of the classical description. The initial singularity, however, is viewed as an attempt to extrapolate the classical theory beyond its natural domain of validity. Near the classical singularity, one expects the evolution of the universe to be governed by a quantum theory of gravity rather than the classical one. Unfortunately, we are yet to formulate a completely satisfactory quantum theory of gravity.

In recent years, the issues regarding singularities in cosmological models have been addressed in a rigorous way within the framework of loop quantum cosmology (LQC) [5-12]. The loop quantum cosmology is a quantization of the cosmological models along the line of a bigger theory known as loop quantum gravity (LQG) [13-16]. It has been shown that the loop quantum cosmology cures the problem of classical singularities in the isotropic model [17] as well as the less symmetric homogeneous model [18], along with quantum suppression of classical chaotic behaviour near singularities in Bianchi-IX models [19, 20]. Further, it has been shown that the non-perturbative modification of the scalar matter Hamiltonian leads to a generic phase of inflation $[21,22]$. These features crucially depend on the fact that the inverse scale operator [23] in loop quantum cosmology has a bounded spectrum. This is in great contrast with the classical situation where the inverse scale factor blows up as the scale factor goes to zero. However, not being a basic operator, quantization of the inverse scale factor operator involves quantization ambiguities [24]. One such ambiguity parameter, referred to as $j$, is related to the dimension of representation of the holonomy operator, and can take any half-integer value (i.e. $j \geqslant \frac{1}{2}$ ). The ambiguity parameter $j$ effectively controls the duration of the LQC-induced inflationary phase. Being an ambiguity parameter, there is no unique way to fix the value of $j$ within loop quantum cosmology itself.

We have mentioned earlier that the density perturbation generated by quantum field fluctuation in an inflating background are believed to be the seed perturbations responsible for the current large-scale structure of the universe. We have also mentioned that nonperturbative modification of the matter Hamiltonian in loop quantum cosmology leads to a generic phase of inflation. Naturally it is an important question to ask whether the density perturbation generated by quantum fluctuations during a loop quantum cosmology induced inflationary phase can satisfy the basic requirements of viability such as a scale-invariant power spectrum. Further, it may leave some distinct imprint on the power spectrum which may be observationally detectable as well.

Being inhomogeneous in nature, treatment of these density perturbations requires inhomogeneous models of loop quantum cosmology. However, the technology required to deal with inhomogeneity at a fundamental level within loop quantum cosmology is not 
available yet. Without such technology, one needs to proceed rather intuitively. Let us recall that in the standard inflationary scenario for computing the power spectrum of the density perturbation due to quantum fluctuations, one uses techniques which can broadly be classified as quantum field theory in a curved background $[25,26]$. In this approach one treats the background geometry as a classical object whereas the matter fields living in it are treated as quantum entities. The main justification for using such techniques comes from the fact that the energy scale associated with the inflationary scenario is a few orders of magnitude lower than the Planck scale. So one expects the geometry to behave more or less classically in this regime.

In loop quantum cosmology, in principle, one can think of using physical observables and physical inner products to evaluate the physical expectation values to find out the behaviour of at least the homogeneous part of the geometrical quantities. Unfortunately developments in physical observables, physical inner products and 'time' evolution in loop quantum cosmology are still at an infant stage [8, 27, 28]. Nevertheless, one can construct an effective but classical description of loop quantum cosmology using WKB techniques. The effective loop quantum cosmology [29] incorporates important non-perturbative modifications and has been shown to be generically non-singular as well [30-32].

In the effective loop quantum cosmology, it has been shown [29] that in the region of interest (exponential inflationary phase) the gravitational part of the Hamiltonian constraint becomes the same as the classical one with small quantum corrections. However, the scalar matter part of the effective Hamiltonian remains non-perturbatively modified during this phase. In fact, non-perturbative modification of the scalar matter Hamiltonian is what that drives inflation in loop quantum cosmology. Having a modified scalar matter Hamiltonian the scalar field satisfies a modified Klein-Gordon equation instead of standard Klein-Gordon equation. Naturally the mode functions of the scalar field, which contain the necessary information about background geometry evolution and are essential to compute the power spectrum of the density perturbations, are expected to be different from the standard mode functions. Thus, although it may be justified to employ similar techniques to compute the power spectrum in effective loop quantum cosmology one certainly cannot borrow the same mode functions used in the standard inflationary scenario.

In this paper, we will compute the power spectrum of the density perturbation using the direct method [33]. In this method, one directly uses the operator expression of the 'time-time' component of the stress-energy tensor (which is classically energy density) to compute the two-point density correlation function and then evaluates its Fourier transform to compute the power spectrum of the density perturbation. In the standard inflationary scenario, one generally avoids this direct computation as the two-point density correlation function in a pure classical background diverges badly for small coordinate length separation (i.e., ultra-violet divergence). There, one usually first computes the power spectrum of the field fluctuation. Using this, one reconstructs the inhomogeneous but classical field configuration which is then used to compute the corresponding density perturbation. However, it is important to understand that this divergence is rather unphysical because it arises when one tries to resolve any two spatial points with arbitrary precision.

In a quantum system, the expectation value of an operator which is classically a phase space function in general is not equal to the same function of the expectation values of basic phase space operators. Thus, the use of the direct method is preferable to the standard method as observational aspects deal with energy density directly rather than the field configuration. Further, in the standard method, one needs to know the general expression of the stress-energy tensor to relate the power spectrum of the field fluctuations to that of the density perturbation. In this context, how to obtain an effective action from a quantum theory of gravity based on canonical quantization is not yet a settled issue. 
In the context of the standard inflationary scenario, it was outlined and explicitly shown in $[33,34]$ that one can in fact regularize this field theoretical divergence by using the notion of zero-point proper length. Although it was used as an ad hoc assertion, it was argued that the notion of zero-point proper length is expected from a proper theory of quantum gravity. The power spectrums of density perturbations computed using these two different methods in the relevant energy scale, however, are not very different. Nevertheless, there one can avoid a rather cumbersome indirect method of computing the power spectrum of density perturbation.

In effective loop quantum cosmology, it has been shown that the universe exhibits a generic big bounce with a non-zero minimum proper volume [32]. This in turn implies a zeropoint proper length for the isotropic spacetime. Since the regularization technique is naturally available in the effective loop quantum cosmology scenario, it is quite appealing to directly use the operator expression of the 'time-time' component of the stress-energy tensor to obtain the power spectrum of the density perturbation due to the quantum field fluctuation. In this sense, this exercise can also be seen as an explicit example of a quantum gravity motivated regularization technique to cure the ultra-violet divergence of standard quantum field theory [35].

In sections 2 and 3, we briefly review the standard scenario of a quantum field living in a de Sitter background and then obtain the corresponding two-point density correlation function. In the following section, we review the scenario of effective loop quantum cosmology which provides the basic infrastructure required to describe its inflationary phase. In particular, we discuss the properties of the effective equation of state for the scalar matter field. The effective equation of state essentially summarizes the evolution of the background geometry. In the following section, we derive the modified Klein-Gordon equation which leads to a modified mode-function equation. We obtain an analytic solution for the mode-function equation. This modified mode function reduces to the standard mode function in the appropriate limit. Using the mode functions in the following section, we compute the power spectrum of the density perturbation. We discuss the properties of the power spectrum and its observational implications.

\section{The quantum field in a de Sitter background}

In computing the power spectrum of the density perturbation in the standard inflationary scenario, one considers the background geometry to be homogeneous and isotropic. The invariant distance element in such spacetime (using natural units, i.e., $c=\hbar=1$ ) is given by the famous Friedmann-Robertson-Walker metric

$$
\mathrm{d} s^{2}=-\mathrm{d} t^{2}+a^{2}(t) \mathrm{d} \mathbf{x}^{2},
$$

where $a(t)$ is the scale factor. During the inflationary period the scale factor grows almost exponentially with coordinate time. The Hubble parameter defined as $H:=\frac{\dot{a}}{a}$ remains almost constant during the period. For simplicity, in the intermediate period of calculation one treats the Hubble parameter as constant, i.e., the evolution of the background geometry is considered to be de Sitter like. One can approximately compute the effect of small variation of the Hubble parameter on the power spectrum simply by considering the variation of the final expression of the power spectrum alone. This is in fact a good approximation as the variation of the Hubble parameter is very small.

In the standard scenario, inflation is driven by a scalar field known as the inflaton field. We will consider here the most simple single-field inflationary scenario. The dynamics of the 
scalar field is governed by the action

$$
S_{\phi}=\int \mathrm{d}^{4} x \sqrt{-g}\left[-\frac{1}{2} g^{\mu \nu} \partial_{\mu} \phi \partial_{\nu} \phi-V(\phi)\right]=\int \mathrm{d}^{4} x \sqrt{-g} \mathcal{L} .
$$

We have mentioned earlier that we will be using the direct method to compute the power spectrum. So it will be quite useful to have an expression for the stress-energy tensor for the scalar field. The stress-energy tensor corresponding to the action (2) is given by

$$
T_{\mu \nu}:=-\frac{2}{\sqrt{-g}} \frac{\delta S_{\phi}}{\delta g^{\mu \nu}}=\partial_{\mu} \phi \partial_{\nu} \phi+g_{\mu \nu} \mathcal{L} .
$$

Comparing with the perfect fluid ansatz, i.e., $T_{\mu \nu}=(\rho+P) u_{\mu} u_{\nu}+g_{\mu \nu} P$, it is easy to see that the $T_{00}$ component represents energy density for the scalar field. In the canonical quantization, one treats the Hamiltonian as a basic object. Thus, it is important for the purpose of this paper to have the expression for the matter Hamiltonian

$$
H_{\phi}=\int \mathrm{d}^{3} x\left[\frac{1}{2} a^{-3} \pi_{\phi}^{2}+\frac{1}{2} a(\nabla \phi)^{2}+a^{3} V(\phi)\right],
$$

where $\pi_{\phi}=a^{3} \dot{\phi}$. In deriving expression (4) it is assumed that the background geometry is homogeneous and isotropic but not the scalar field itself. This approximation can be justified as long as the deviation from the homogeneity and isotropy remains small. To make it more clear, we rewrite the scalar field Hamiltonian (4) as

$$
H_{\phi}=a^{-3} \int \mathrm{d}^{3} x\left[\frac{1}{2} \pi_{\phi}^{2}\right]+a \int \mathrm{d}^{3} x\left[\frac{1}{2}(\nabla \phi)^{2}\right]+a^{3} \int \mathrm{d}^{3} x[V(\phi)] .
$$

In loop quantum cosmology, the geometrical quantities such as the scale factor $a$ are represented here through corresponding quantum operators. While deriving effective classical Hamiltonian from loop quantum cosmology, these operator expressions effectively get replaced by their corresponding eigenvalues. The kinetic term of the scalar matter Hamiltonian (5) involves inverse powers of the scale factor. In loop quantum cosmology, the inverse scale factor operator has a bounded spectrum. Clearly one can see that the kinetic term of the effective scalar matter Hamiltonian will involve non-perturbative modifications due to loop quantization. Using Hamilton's equations of motion for the scalar field, i.e.

$$
\dot{\phi}=\frac{\delta H_{\phi}}{\delta \pi_{\phi}} ; \quad \dot{\pi}_{\phi}=-\frac{\delta H_{\phi}}{\delta \phi},
$$

one can derive the second-order equation of motion for the scalar field, given by

$$
\ddot{\phi}+3\left(\frac{\dot{a}}{a}\right) \dot{\phi}-\frac{\nabla^{2} \phi}{a^{2}}+V^{\prime}(\phi)=0 .
$$

The equation of motion (7) for the scalar field is the standard Klein-Gordon equation. It is worthwhile emphasizing that one could have obtained the standard Klein-Gordon equation (7) simply by considering the variation of the scalar field action (2). But one should remember that our ultimate aim is to compute power spectrum in effective loop quantum cosmology where non-perturbative modification in the matter sectors comes through its Hamiltonian.

To quantize the scalar field one proceeds in the standard way, i.e., by decomposing scalar field operator in terms of annihilation and creation operators $\hat{a}$ and $\hat{a^{\dagger}}$ as follows:

$$
\hat{\phi}(\mathbf{x}, t)=\int \frac{\mathrm{d}^{3} \mathbf{k}}{(2 \pi)^{3}}\left[\hat{a}_{k} f_{k}(t) \mathrm{e}^{\mathrm{i} \mathbf{k} \cdot \mathbf{x}}+\hat{a}_{k}^{\dagger} f_{k}^{*}(t) \mathrm{e}^{-\mathrm{ik} \cdot \mathbf{x}}\right],
$$

where $f_{k}(t)$ are the 'properly normalized' mode functions. Although one can quantize the scalar field analogous to that in Minkowski spacetime, one faces the well-known problem of 
defining the vacuum state in curved spacetime. In general, for a curved background geometry a unique choice for the vacuum state does not exist. Thus, one needs to have some additional prescription to define it.

In the standard inflationary scenario one generally chooses the so-called Bunch-Davies vacuum. It is defined as the state which gets annihilated by $\hat{a}$ where the mode functions $f_{k}$ are so 'normalized' such that in the 'Minkowskian limit', i.e., $H \rightarrow 0$, the mode function reduces to the flat-space positive frequency mode function $\frac{1}{\sqrt{2 \omega}} \mathrm{e}^{-\mathrm{i} \omega t}$. We will use an analogous definition for the vacuum state for the calculation of the power spectrum in effective loop quantum cosmology as well. For simplicity, we consider the situation where the field potential is made of only the mass term (i.e., $V(\phi)=\frac{1}{2} m_{\phi}^{2} \phi^{2}$ ). Then the mode functions are the solution of the equation

$$
\ddot{f}_{k}+3\left(\frac{\dot{a}}{a}\right) \dot{f}_{k}+\left(\frac{k^{2}}{a^{2}}+m_{\phi}^{2}\right) f_{k}=0 .
$$

The mode-function equation (9) follows from the Klein-Gordon equation (7) and the expansion of the scalar field operator (8). For simplicity, we consider the situation where the mass term can be neglected $\left(\frac{k}{a} \gg m_{\phi}\right)$ in equation (9). The 'normalized' mode-function solutions are then given by

$$
f_{k}=\frac{H}{\sqrt{2 k^{3}}}\left(1-\mathrm{i} \frac{k}{H a}\right) \mathrm{e}^{\mathrm{i} \frac{k}{H a}} .
$$

The mode function (10) in the 'Minkowskian limit', i.e., $H \rightarrow 0$, reduces (up to a constant phase) to the flat-space positive frequency mode function $\frac{1}{\sqrt{2 \omega}} \mathrm{e}^{-\mathrm{i} \omega t}$. This defines the vacuum state $|0\rangle$ as $\hat{a}|0\rangle=0$.

To compute the power spectrum of the density perturbation using the indirect method, one first computes the power spectrum of field fluctuation, i.e., $\mathcal{P}_{\phi}(k):=\frac{k^{3}}{2 \pi^{2}}\left|f_{k}\right|^{2}$. It is easy to see from the expression of the normalized mode function (10) that at the time of horizon crossing $\left(a(t)=\frac{k}{2 \pi H}\right)$, the corresponding power spectrum is scale invariant. In getting the mode-function solution (10), we have ignored the mass term of the scalar field. For the mass dominating case $\left(\frac{k}{a} \ll m_{\phi}\right)$, the 'normalized' mode functions are

$$
f_{k}=\frac{1}{\sqrt{2 m_{\phi}}} a^{-\frac{3}{2}} \mathrm{e}^{-\mathrm{i} m_{\phi} t\left(\sqrt{1-\left(\frac{3 H}{2 m_{\phi}}\right)^{2}}\right)} .
$$

It can be easily checked that for this case also the corresponding power spectrum is scale invariant at the time of horizon crossing. It is often argued that the scale invariance is mainly determined by the fact that during the inflationary period, the Hubble horizon $H^{-1}$ remains almost constant. The detail of this particular model of inflation have rather small effect on this property of the power spectrum.

\section{The two-point density correlation function}

Having specified the vacuum state, one can proceed to evaluate the vacuum expectation value of the two-point density correlation function. The two-point density correlation function can naturally be defined as

$$
C(\mathbf{x}+\mathbf{l}, \mathbf{x}, t):=\left\langle 0\left|\hat{T}_{0}^{0}(\mathbf{x}+\mathbf{l}, t) \hat{T}_{0}^{0}(\mathbf{x}, t)\right| 0\right\rangle .
$$


Using the expression of the scalar field operator (8) and the expression of the stress-energy tensor (3), one can evaluate the two-point density correlation function in terms of the mode function, given by [33]

$$
C(\mathbf{x}+\mathbf{l}, \mathbf{x}, t)=\int \frac{\mathrm{d}^{3} \mathbf{p}}{(2 \pi)^{3}} \frac{\mathrm{d}^{3} \mathbf{q}}{(2 \pi)^{3}} \mathrm{e}^{\mathrm{i}(\mathbf{p}+\mathbf{q}) \cdot \mathbf{l}}\left|\dot{f}_{p} \dot{f}_{q}-\left(\frac{\mathbf{p} \cdot \mathbf{q}}{a^{2}}-m_{\phi}^{2}\right) f_{p} f_{q}\right|^{2} .
$$

In evaluating two-point density correlation function (12), one ignores a space-independent (formally divergent) term as it would have contributed only to the $k=0$ mode while taking a Fourier transform. Knowing the normalized mode-function solution $f_{k}(10)$, one can explicitly calculate the two-point density correlation function (12) given by [33]

$$
C\left(l^{\prime}, t\right):=C(\mathbf{x}+\mathbf{l}, \mathbf{x}, t)=\frac{1}{4 \pi^{4}}\left[\frac{2 H^{2}}{\left(a l^{\prime}\right)^{6}}+\frac{12}{\left(a l^{\prime}\right)^{8}}\right],
$$

where $l^{\prime}=|\mathbf{I}|$. Expression (13) of the two-point density correlation function expectedly diverges near $l^{\prime}=0$. However, as shown in [33], one can regularize this divergence using the notion of zero-point proper length. Expression (13) in 'Minkowskian limit', i.e., $H \rightarrow 0$, reduces to the flat-space two-point density correlation function.

\section{Effective isotropic loop quantum cosmology}

In isotropic loop quantum cosmology, the basic phase space variables are Ashtekar connection $c$ and densitized triad $p$. The geometrical property of the space is encoded in the densitized triad $p$ whereas the dynamics is encoded in the connection $c$. In loop quantum cosmology, one redefines the densitized triad to absorb the fiducial coordinate volume component. This makes the proper volume of the universe (1), $\int \mathrm{d}^{3} x \sqrt{-g}=a^{3} V_{0}=p^{\frac{3}{2}}$ [12].

In loop quantum cosmology, the development of physical observables and the physical inner product are still at an infant stage. Nevertheless, one can derive via the WKB method, an effective classical Hamiltonian which incorporates the most important non-perturbative modifications. This allows one to study the effects of quantum modification using available standard tools. The effective Hamiltonian for spatially flat isotropic loop quantum cosmology derived in [29] is given by

$$
H^{\mathrm{eff}}=-\frac{1}{\kappa} \frac{B_{+}(p)}{4 p_{0}} K^{2}+W_{q g}+H_{\phi}^{\mathrm{eff}}
$$

where $\kappa=16 \pi G, p_{0}=\frac{1}{6} \gamma \ell_{\mathrm{P}}^{2} \mu_{0}, \gamma$ is the Barbero-Immirzi parameter, $K$ is the extrinsic curvature (conjugate variable of $p), A(p)=\left|p+p_{0}\right|^{\frac{3}{2}}-\left|p-p_{0}\right|^{\frac{3}{2}}, B_{+}(p)=A\left(p+4 p_{0}\right)+A$ $\left(p-4 p_{0}\right), \ell_{\mathrm{P}}^{2}:=\kappa \hbar$ and $W_{q g}=\left(\frac{\ell_{\mathrm{P}}^{4}}{288 \kappa p_{0}^{3}}\right)\left\{B_{+}(p)-2 A(p)\right\} . \mu_{0}$ here is viewed as a quantization ambiguity parameter and it is an order one number $[12,16]$. Apart from the modifications of the gravitational kinetic term and scalar matter kinetic term, the effective Hamiltonian (14) differs from the classical Hamiltonian by a non-trivial potential term referred to as quantum geometry potential $W_{q g}$. In this paper, we are interested in the regime $p_{0} \ll p$ where the quantum geometry potential has the natural interpretation of being perturbative homogeneous quantum fluctuations around a FRW background. The effective scalar matter Hamiltonian is given by

$$
H_{\phi}^{\mathrm{eff}}=\frac{1}{2}\left|\tilde{F}_{j, l}(p)\right|^{\frac{3}{2}} p_{\phi}^{2}+p^{\frac{3}{2}} V(\phi),
$$

where $p_{\phi}\left(=V_{0} \pi_{\phi}\right)$ is the field momentum, $\tilde{F}_{j, l}(p)$ is the eigenvalue of the inverse densitized triad operator $p^{-1}$ and is given by $\tilde{F}_{j, l}(p)=\left(p_{j}\right)^{-1} F_{l}\left(p / p_{j}\right)$ where $p_{j}=\frac{1}{3} \gamma \mu_{0} j l_{p}^{2}$. The $j$ and $l$ are two quantization ambiguity parameters $[24,36]$. The half integer $j$ is related with 
the dimension of representation while writing holonomy as multiplicative operators. The real valued $l(0<l<1)$ corresponds to different, classically equivalent, ways of writing the inverse power of the densitized triad in terms of the Poisson bracket of the basic variables. The function $F_{l}(q)$ is given by [20]

$$
\begin{aligned}
F_{l}(q):= & {\left[\frac { 3 } { 2 ( l + 2 ) ( l + 1 ) l } \left((l+1)\left\{(q+1)^{l+2}-|q-1|^{l+2}\right\}\right.\right.} \\
& \left.\left.-(l+2) q\left\{(q+1)^{l+1}-\operatorname{sgn}(q-1)|q-1|^{l+1}\right\}\right)\right]^{\frac{1}{1-l}} \\
\rightarrow & q^{-1} \quad(q \gg 1) \\
\rightarrow & {\left[\frac{3 q}{l+1}\right]^{\frac{1}{1-l}} \quad(0<q \ll 1) . }
\end{aligned}
$$

From expression (16) one should note that for large values of the densitized triad, i.e., in a large volume, one has the expected classical behaviour for the inverse densitized triad. The quantum behaviour is manifested for smaller values of the densitized triad. Here, the meaning of large or small values of the triad $p$ is determined necessarily by the values of $p_{j}$. The quantum mechanically allowed values for the ambiguity parameter $l$ are $0<l<1$. Now one should also note that if one takes the ambiguity parameter value $l=2$ then the small volume expression (16) becomes the same as the large volume expression. In other words, taking ambiguity parameter value $l=2$ is equivalent to taking the large volume limit, i.e. the classical limit. This observation will be very useful in fixing the choice of vacuum while computing the two-point density correlation function in this effective background.

In loop quantum cosmology, $p_{0}$ and $p_{j}$ represent two important (square of) length scales: $p_{0}$ demarcates the strong quantum effect regime (non-perturbative regime) from the weak quantum effect regime (perturbative regime) of the gravity sector whereas $p_{j}$ demarcates the same for the matter sector. Since ambiguity parameter $j \geqslant \frac{1}{2}$, it follows from their respective definition that $p_{j} \geqslant p_{0}$. Naturally, non-perturbative modification of matter sector can survive longer than the same for the gravity sector depending on the value of the ambiguity parameter $j$. We have mentioned earlier that in computing the power spectrum of the density perturbation we will use similar techniques to those used in the standard inflationary scenario. In this approach one treats geometry as a classical object whereas matter fields living in it are treated as quantum objects. Thus, self-consistency of this framework requires that we should consider the regime where $p \gg p_{0}$ in our calculation. In this regime, the gravitational part of the Hamiltonian constraint becomes the same as the classical Hamiltonian with a small quantum correction. The reduced effective Hamiltonian in this regime is given by

$$
H^{\mathrm{eff}}=-\frac{3}{2 \kappa} K^{2} \sqrt{p}-\frac{\ell_{\mathrm{P}}^{4}}{24 \kappa} p^{-\frac{3}{2}}+H_{\phi}^{\mathrm{eff}}
$$

The loop quantum cosmology induced inflationary scenario persist as long as the densitized triad $p$ remains less than $p_{j}$. Thus, we will be interested in computing the power spectrum of the density perturbation in the regime $p_{0} \ll p<p_{j}$. In this regime, the effective energy density and pressure are given by $\rho^{\text {eff }}=p^{-\frac{3}{2}} H_{\phi}^{\text {eff }}$ and $P^{\text {eff }}=-\frac{1}{3} p^{-\frac{3}{2}}\left(2 p \frac{\partial H_{\phi}^{\text {eff }}}{\partial p}\right)$ [29]. It can be checked easily using the relation between scale factor and densitized triad that these definitions satisfy the standard conservation equation $a \frac{\mathrm{d} \rho^{\mathrm{eff}}}{\mathrm{d} a}=-3\left(\rho^{\mathrm{eff}}+P^{\mathrm{eff}}\right)$. Furthermore, one can recover the standard expression of energy density and pressure using the standard scalar matter Hamiltonian $H_{\phi}$ in place of the modified scalar matter Hamiltonian $H_{\phi}^{\text {eff }}$ in these 
definitions. It is shown in [22] that the effective equation of state $\omega^{\text {eff }}:=P^{\text {eff }} / \rho^{\text {eff }}$ can be expressed as a function of the standard equation of state $\omega$ and the densitized triad $p$

$$
\omega^{\text {eff }}=-1+\frac{(1+\omega) p^{\frac{3}{2}}\left[\tilde{F}_{j, l}(p)\right]^{\frac{3}{2}}\left(1-\frac{p}{\tilde{F}_{j, l}(p)} \frac{\mathrm{d} \tilde{F}_{j, l}(p)}{\mathrm{d} p}\right)}{(1+\omega) p^{\frac{3}{2}}\left[\tilde{F}_{j, l}(p)\right]^{\frac{3}{2}}+(1-\omega)} .
$$

Using expression (16), it is easy to see that for the large values of the densitized triad $p$, where one expects the quantum effects to be small, $\omega^{\text {eff }}=\omega$, whereas for small values of $p$ the $\omega^{\text {eff }}$ differs from the classical $\omega$ dramatically. In this paper, we will be interested in the situation where $\omega^{\text {eff }} \approx-1$ (for $p<p_{j}$ ). This requirement will automatically be satisfied if at the end of loop quantum cosmology induced inflation the radiation or matter domination or even another phase of classical acceleration (i.e., $\omega=\frac{1}{3}, 0,<-\frac{1}{3}$ ) begins. Thus, during the loop quantum cosmology induced inflationary period, one can express the matter Hamiltonian as

$$
H_{\phi}^{\mathrm{eff}} \approx \bar{\rho} p^{\frac{3}{2}}
$$

where $\bar{\rho}$ is a constant of integration. Physically, $\bar{\rho}$ corresponds to the maximum energy density that can be 'stored' in the effective spacetime. This also defines the energy scale associated with the loop quantum cosmology induced inflationary scenario.

It has been shown in [32] that the effective loop quantum cosmology exhibits a generic bounce with non-zero minimum proper volume. It follows from equations (17) and (19) that the minimum value of the proper distance $L_{0}$, defined as $L_{0}^{2}:=p_{\min }=p\left(H^{\mathrm{eff}}=0 ; K=0\right)$, is given by

$$
L_{0}^{6}=\frac{2 \pi G}{3 \bar{\rho}} .
$$

Self-consistency of the expression (20) requires $p_{0} \ll p_{\min }<p_{j}$.

In standard cosmology one uses the scale factor as a geometric variable. In isotropic loop quantum cosmology, the basic variable is the densitized triad $p$ defined as $p^{\frac{3}{2}}:=\int \mathrm{d}^{3} x \sqrt{-g}=$ $a^{3} V_{0}$, where $V_{0}$ is the fiducial coordinate volume. Clearly, the densitized triad $p$ here is a dimensionful quantity whereas the scale factor $a$ is dimensionless. Also the absolute value of the scale factor is physically irrelevant. Rather, what matters is the ratio of scale factor at two different periods. Naturally, there is a freedom left in relating the scale factor with the densitized triad. We define the relation between the scale factor and the eigenvalues of the densitized triad operator such that for a small volume limit

$$
\hat{p} \hat{p}^{-1}|\mu\rangle:=a^{2\left(1+\frac{1}{1-l}\right)}|\mu\rangle .
$$

We have mentioned earlier that taking the ambiguity parameter value $l=2$ is equivalent to taking a large volume limit of the inverse densitized triad spectrum. Clearly, in our choice of definition the scale factor takes the value $a=1$ at the transition point from the nonperturbatively modified matter sector to the standard matter sector. For the regime $p<p_{j}$, one can approximate the effective equation of state (18) as

$$
\left(1+\omega^{\mathrm{eff}}\right) \simeq C_{\omega}\left(\frac{n+2}{3}\right) a^{2(1-n)},
$$

where $C_{\omega}=2\left(\frac{1+\omega}{1-\omega}\right)$ and $n=-\frac{1}{2}\left(1+\frac{3}{1-l}\right)$. The last two terms in the effective Hamiltonian constraint (17) are comparable near the bounce point. However, once the densitized triad $p$ starts increasing then it is clear from equation (17) that the contribution from quantum geometry potential quickly drops out compared to the matter Hamiltonian (19). Naturally for the region away from the bounce point, one can write the Hamiltonian constraint $\left(H^{\text {eff }}=0\right)$ 
in terms of the scale factor as

$$
3\left(\frac{\dot{a}}{a}\right)^{2} \simeq 8 \pi G \bar{\rho},
$$

where we have used Hamilton's equation of motion $\dot{p}=\frac{\kappa}{3} \frac{\partial H^{\text {eff }}}{\partial K}$. Equation (23) is nothing but the usual Friedmann equation. Using equations (20) and (23), we can define a dimensionless quantity

$$
\sigma:=2 \pi H L_{0}=4 \pi\left(\frac{2 \pi}{3}\right)^{\frac{2}{3}}\left(\frac{\bar{\rho}}{M_{p}^{4}}\right)^{\frac{1}{3}},
$$

where $G=M_{p}^{-2}$. This will be a useful quantity in the calculation of the power spectrum. Since we consider the situation where $p_{0} \ll L_{0}^{2}<p_{j}$, i.e., the bounce occurs at a time when the proper volume of the universe is much larger than the Planck volume. Thus, it is clear that $\sigma$ is much smaller than unity $(\sigma \ll 1)$ during the loop quantum cosmology induced exponential inflationary phase.

From the definition of the FRW metric (1), it follows that the proper distance square, say $d^{2}\left(a, l^{\prime}\right)$, between two points separated by coordinate distance $l^{\prime}$ on a given spatial slice $(d t=0)$ is simply $d^{2}\left(a, l^{\prime}\right)=\left(a l^{\prime}\right)^{2}$. In other words, in the classical geometry the proper distance between two points is simply 'coordinate distance times the scale factor'. In the classical case one can choose the coordinate distance separation to be arbitrarily small. Naturally, the proper distance between two points can become arbitrarily small. In loop quantum cosmology, the basic variable is a densitized triad instead of the usual metric variable. Further, in loop quantum cosmology, one redefines the densitized triad by absorbing a component of the fiducial coordinate volume. This makes the proper volume of the universe just $p^{\frac{3}{2}}$. In the case of effective loop quantum cosmology, we have seen that there exists a non-zero minimum value for the densitized triad $p$. To incorporate such a feature in the definition of the proper distance in effective loop quantum cosmology, we introduce the notion of effective coordinate length $l^{\mathrm{eff}}\left(a, l^{\prime}\right)$. The proper distance between two points separated by coordinate distance $l^{\prime}$ is defined as

$$
d^{2}\left(l^{\prime}, a\right):=\left(a l^{\mathrm{eff}}\right)^{2}=L_{0}^{2}+\left(a l^{\prime}\right)^{2}
$$

The effective coordinate length keeps the usual notion of proper distance, i.e., 'coordinate distance times scale factor', intact and incorporates features such as zero-point proper length. Further, it allows one to use the standard machinery while computing the power spectrum of the density perturbation and acts as an ultra-violet regulator of standard quantum field theory. For large volumes (i.e., $\left(a l^{\prime}\right)$ large), this definition is virtually equivalent to the standard definition of proper distance as $L_{0}$ is very small (a few Planck units).

\section{The modified Klein-Gordon equation}

We mentioned earlier that the kinetic term of the scalar matter Hamiltonian gets nonperturbative modification as its classical expression involves inverse powers of the densitized triad. The effective scalar matter Hamiltonian obtained, as outlined in the previous section, is given by

$H_{\phi}^{\mathrm{eff}}=V_{0}\left|\tilde{F}_{j, l}(p)\right|^{\frac{3}{2}} \int \mathrm{d}^{3} x\left[\frac{1}{2} \pi_{\phi}^{2}\right]+V_{0}^{-\frac{1}{3}} p^{\frac{1}{2}} \int \mathrm{d}^{3} x\left[\frac{1}{2}(\nabla \phi)^{2}\right]+V_{0}^{-1} p^{3 / 2} \int \mathrm{d}^{3} x[V(\phi)]$.

It should be noted that we have now kept the gradient term in the effective Hamiltonian. Earlier, while computing the background evolution the gradient term was neglected as one 
assumes that the background evolution is mainly determined by the homogeneous and isotropic contribution of the matter Hamiltonian. In other words, the inhomogeneity is assumed to be small. Using the Hamilton equations of motion for the effective Hamiltonian (26), one can derive the corresponding modified Klein-Gordon equation, given by

$$
\ddot{\phi}-3\left(\frac{1}{1-l}\right)\left(\frac{\dot{a}}{a}\right) \dot{\phi}+a^{3+\frac{3}{1-l}}\left(-\frac{\nabla^{2} \phi}{a^{2}}+V^{\prime}(\phi)\right)=0,
$$

where we have substituted the eigenvalue of the inverse triad operator by the scale factor using definition (21). It is easy to see that if one takes the value of the ambiguity parameter $l=2$ then the modified Klein-Gordon equation (27) goes back to the standard Klein-Gordon equation (7).

It is important to emphasize here that the non-perturbative modification of the scalar matter Hamiltonian, which is being studied here, comes from the bounded spectrum of the inverse scale factor operator. Since the modification affects the kinetic term of the scalar matter Hamiltonian, it essentially affects all the modes. It can be seen from equation (27) as well. This modification is distinct from other Planck-scale effects studied in the literature. For example, in the context of trans-Planckian inflation [43, 44], one studies the possible effects of Planck-scale modification of the dispersion relation or the possible effects of the spacetime non-commutativity [45, 46].

\section{Modified mode functions}

Using the expression for the quantized scalar field (8) as in the standard case, one can derive the modified mode-function equation for the scalar field

$$
\ddot{f}_{k}-3\left(\frac{1}{1-l}\right)\left(\frac{\dot{a}}{a}\right) \dot{f}_{k}+a^{3+\frac{3}{1-l}} H^{2}\left(\frac{k^{2}}{H^{2} a^{2}}+\frac{m_{\phi}^{2}}{H^{2}}\right) f_{k}=0 .
$$

One can easily check that for $l=2$ (i.e., the classical case) the modified mode-function equation goes back to the standard mode-function equation (9). To compute the power spectrum, it is essential to know the solution of the mode-function equation (28). For simplicity, we will neglect the mass term, i.e., we will assume $\frac{k}{\mathrm{Ha}} \gg \frac{m_{\phi}}{\mathrm{H}}$. To simplify the mode-function equation (28) further, we make a change of variables as follows:

$$
f_{k}:=a^{-n} \tilde{f}_{k} ; \mathrm{d} t=a^{n} \mathrm{~d} \eta
$$

with the value of $n=-\frac{1}{2}\left(1+\frac{3}{1-l}\right)$. In loop quantum cosmology allowed values for the $l$ ambiguity parameter are in the range $0<l<1$ whereas the classical situation can be obtained simply by taking $l=2$. In terms the new parameter $n$, the classical situation corresponds to $n=1$ and the quantum situation is described for $-\infty<n<-2$. One may note here that for $n=1$ the new variable $\eta=-\frac{1}{n H a^{n}}$ is nothing but conformal time. In terms of these new variables (29), the mode-function equation (28) becomes

$$
\frac{\mathrm{d}^{2} \tilde{f}_{k}}{\mathrm{~d} \eta^{2}}-\left(2 \frac{1}{2 n}-1\right) \frac{1}{\eta} \frac{\mathrm{d} \tilde{f}_{k}}{\mathrm{~d} \eta}+\left(k^{2}+\frac{\left(\frac{1}{2 n}\right)^{2}-\left(1+\frac{1}{2 n}\right)^{2}}{\eta^{2}}\right) \tilde{f}_{k}=0
$$

Equation (30) is a modified expression of the Bessel differential equation and admits an analytical solution of the form [37]

$$
\tilde{f}_{k}=\eta^{\frac{1}{2 n}}\left[A_{(k, n)} J_{-\left(1+\frac{1}{2 n}\right)}(k \eta)+B_{(k, n)} J_{\left(1+\frac{1}{2 n}\right)}(k \eta)\right],
$$

where $A_{(k, n)}$ and $B_{(k, n)}$ are two constants of integration corresponding to the second-order differential equation of the mode function. 
To fix these constants of integration, we require that for large volumes $(n=1)$ the modified mode function reduces to the standard 'normalized' mode function (10). Since the standard mode function (10) is already 'normalized' to pick out the Bunch-Davies vacuum then this requirement will automatically fix the choice of vacuum in effective loop quantum cosmology. This fixes the mode-function solution as

$$
f_{k}=\sqrt{\frac{n+2}{3}}(-n H) \sqrt{\frac{\pi}{4 k^{3}}}(k \eta)^{1+\frac{1}{2 n}}\left[J_{-\left(1+\frac{1}{2 n}\right)}(k \eta)+\mathrm{i} J_{\left(1+\frac{1}{2 n}\right)}(k \eta)\right] .
$$

Using Bessel function identities $J_{n+1}(x)+J_{n-1}(x)=\frac{2 n}{x} J_{n}(x), J_{-\frac{1}{2}}=\sqrt{\frac{2}{\pi x}} \cos (x)$ and $J_{\frac{1}{2}}=\sqrt{\frac{2}{\pi x}} \sin (x)$, one can easily check that for $n=1$ the modified mode function (32) reduces to the standard mode function (10).

It is worth pointing out that in expression (32), we have specifically chosen the power of $\left(\frac{n+2}{3}\right)$ to be $\frac{1}{2}$. But it is clear that for any arbitrary power of $\left(\frac{n+2}{3}\right)$, the mode function would reduce to the standard mode function. We have made this choice precisely to absorb a similar term coming from the effective equation of state (22) that appears in the final expression of power spectrum. In other words, we have chosen the vacuum state such that it satisfies the Bunch-Davies prescription and the computed power spectrum is free from trivial ambiguity parameter dependent multiplicative factors.

\section{The power spectrum}

Knowing the exact solution of the mode function in principle, one can evaluate the two-point density correlation function using expression (12). Let us recall that we are mainly interested in finding the power spectrum at the time of horizon crossing, i.e., $\frac{k}{H a}=2 \pi$. The argument of the Bessel function $k \eta=\frac{k}{H a}\left(\frac{a^{1-n}}{-n}\right) \ll 1$ during horizon crossing. For the super-horizon scale the above inequality holds naturally; even for the sub-horizon scale up to reasonable extent the same inequality will hold since for effective loop quantum cosmology $-\infty<n<-2$. Since the asymptotic form of the Bessel function is $J_{m}(x) \approx \frac{1}{\Gamma(1+m)}\left(\frac{x}{2}\right)^{m}$ for $x \ll 1$ then clearly the dominating contribution in the mode function (32) comes from the first term. So we will approximate the mode function as

$$
f_{k} \approx \sqrt{\frac{n+2}{3}}(-n H) \sqrt{\frac{\pi}{4 k^{3}}}(k \eta)^{1+\frac{1}{2 n}}\left[J_{-\left(1+\frac{1}{2 n}\right)}(k \eta)\right]
$$

for further evaluation and will use its asymptotic form for explicit calculation.

Using the expression for the two-point density correlation function (12) and the expression for the mode function (33), one can simply follow similar steps as in [33] to derive the expression of the two-point density correlation function

$$
C\left(l^{\prime}, t\right)=\left(\frac{n+2}{3}\right)^{2} \frac{a^{4(1-n)}}{2^{2-\frac{2}{n}}(2 \pi)^{2} \Gamma\left(1-\frac{1}{2 n}\right)^{4}}\left[\frac{2 H^{2}}{\left(a l^{\prime}\right)^{6}}+\frac{a^{4(1-n)}}{\left(a l^{\prime}\right)^{8}}\right] .
$$

In deriving the above expression (34), it is quite helpful to use the Bessel function identity $\frac{\mathrm{d}}{\mathrm{d} x}\left[x^{m} J_{-m}(x)\right]=-x^{m} J_{1-m}(x)$ while evaluating the time derivative of the mode function (33). Now it is easy to check that for $n=1$ (i.e., classical mode function), the expression becomes qualitatively the same as (13). But we may note that it is quantitatively slightly different from (13). The source of this difference can be traced back to the approximation that we have made. In the case of loop quantum cosmology, the argument of the Bessel function $k \eta=\frac{k}{H a}\left(\frac{a^{1-n}}{-n}\right) \ll 1$ at the time of horizon crossing. But clearly the same does not hold for the standard case $(n=1)$. 
The two-point density correlation function (34) diverges as the 'coordinate length' $l$ ' goes to zero. This feature is expected from a calculation based on standard quantum field theory. However, one can regularize this expression using the notion of zero-point proper length which is naturally available in effective loop quantum cosmology. In section 4 , we have introduced the notion of effective coordinate length. This basically allows one to use the machinery of the standard case. Essentially this step summarizes the ultra-violet regularization of the two-point density correlation function. We define effective two-point density correlation function as the regularized form of the standard two-point density correlation function as

$$
C^{\mathrm{eff}}\left(l^{\prime}, t\right):=C\left(l^{\mathrm{eff}}, t\right) \text {. }
$$

Now we can evaluate the Fourier transform of the effective two-point density correlation in the usual way:

$$
\begin{aligned}
\left|\rho_{k}(t)\right|^{2} & :=\int \mathrm{d}^{3} \mathbf{l} \mathrm{e}^{\mathrm{i} \cdot \mathbf{l}} C^{\mathrm{eff}}\left(l^{\prime}, t\right) \\
& =\left(\frac{n+2}{3}\right)^{2} \frac{a^{4(1-n)}}{2^{2-\frac{2}{n}}(2 \pi)^{2} \Gamma\left(1-\frac{1}{2 n}\right)^{4}}\left[\frac{2 H^{2}}{a^{6}} I_{1}+\frac{a^{4(1-n)}}{a^{8}} I_{2}\right],
\end{aligned}
$$

where the integrals $I_{1}$ and $I_{2}$ can be evaluated using the method of contour integration. They are given by

and

$$
I_{1}:=\int \frac{\mathrm{d}^{3} \mathbf{I} \mathrm{e}^{\mathrm{i} \mathbf{k} \cdot \mathbf{l}}}{\left(l^{2}+\frac{L_{0}^{2}}{a^{2}}\right)^{3}}=\frac{\pi^{2} \mathrm{e}^{-\frac{k L_{0}}{a}}}{4}\left(\frac{a}{L_{0}}\right)^{3}\left[1+\frac{k L_{0}}{a}\right],
$$

$$
I_{2}:=\int \frac{\mathrm{d}^{3} \mathbf{I} \mathrm{e}^{\mathrm{ik} \cdot \mathbf{l}}}{\left(l^{2}+\frac{L_{0}^{2}}{a^{2}}\right)^{4}}=\frac{\pi^{2} \mathrm{e}^{-\frac{k L_{0}}{a}}}{8}\left(\frac{a}{L_{0}}\right)^{5}\left[1+\frac{k L_{0}}{a}+\frac{1}{3}\left(\frac{k L_{0}}{a}\right)^{2}\right] .
$$

The power spectrum of the density perturbation generated during inflation, however, is not directly observable. Rather, the observed power spectrum corresponds to the density perturbation at the time of horizon re-entry in the post-inflationary period. In the intermediate period between horizon exit and horizon re-entry the density contrast $\delta\left(:=\frac{\delta \rho}{\rho}\right)$ remains almost constant for the super-horizon modes. Nevertheless, the change in the equation of state of the total matter field leads to a scaling of the amplitude of the density perturbation. Super-horizon evolution in Bardeen's gauge-invariant formalism [38] of the density perturbation leads to a rather simple formula for the evolution of density contrast:

$$
\left|\frac{\delta_{k}}{1+\omega}\right|_{t=t_{f}} \approx\left|\frac{\delta_{k}}{1+\omega}\right|_{t=t_{i}},
$$

where $\delta_{k}:=\frac{\rho_{k}(t)}{\bar{\rho}}, t_{i}$ and $t_{f}$ are initial and final time, respectively. The power spectrum of the density perturbation at the time of re-entry is given by

$$
\mathcal{P}_{\delta}(k)=\frac{k^{3}}{2 \pi^{2}}\left|\delta_{k}\right|_{\text {re-entry }}^{2}=\mathcal{A}^{2}\left[1+c_{0}\left(\frac{k}{2 \pi H}\right)^{4(1-n)}\right]
$$

where $c_{0}=\frac{\pi^{2}}{\sigma^{2}}\left[1+\frac{\sigma^{2}}{3(1+\sigma)}\right]$ and the $\mathcal{A}^{2}$ is given by

$$
\mathcal{A}^{2}=\frac{\left(1+\omega_{\mathrm{re}}\right)^{2}}{(2 \pi)^{2} C_{\omega}^{2}} \frac{\sigma^{3}(1+\sigma) \mathrm{e}^{-\sigma}}{2^{2-\frac{2}{n}} \Gamma\left(1-\frac{1}{2 n}\right)^{4}} .
$$

The quantity $\sigma$, defined in (24), is given by $\sigma=4 \pi\left(\frac{2 \pi}{3}\right)^{\frac{2}{3}}\left(\frac{\bar{\rho}}{M_{p}^{4}}\right)^{\frac{1}{3}}$. For the super-horizon modes $\left(\frac{k}{2 \pi H} \ll 1\right)$, the second term in expression (40) is negligible compared to unity for the effective 
loop quantum cosmology $(-\infty<n<-2)$. Thus, it is clear from expression (40) that power spectrum of the density perturbation is broadly scale invariant as during the inflationary period the Hubble parameter remains almost constant. Since the mode-function solutions (32) are ambiguity parameter dependent, we expect the expression of the power spectrum (40) to depend on ambiguity parameter. But it should also be noted that this dependence is rather weak. The ambiguity parameter dependent term in the power spectrum $2^{-\frac{2}{n}} \Gamma\left(1-\frac{1}{2 n}\right)^{4}$ varies only between 1 and 1.3499 for the range of ambiguity parameter value $(-\infty<n<-2)$.

An important property of the power spectrum (40) is that $\mathcal{A}^{2} \sim H^{2}$ ( $\sigma$ being small $\left.(1+\sigma) \mathrm{e}^{-\sigma} \approx 1\right)$. This behaviour is exactly similar to the behaviour of the power spectrum in the standard inflationary scenario. This property of the power spectrum will be very useful in comparison to the spectral index between the standard inflationary scenario and the effective loop quantum cosmology scenario.

\subsection{The amplitude of the density perturbation}

In section 4, we have shown that the self-consistency of the framework that we are using requires $\sigma \ll 1$. This requirement can be physically understood in the following way. The effective continuum (classical geometrical) description is an emergent description in the loop quantum cosmology framework in which underlying geometry is fundamentally discrete. Naturally, the effective Hamiltonian description which has been used in the paper has a restricted domain. In [32], it has been shown that the dynamics described by the effective Hamiltonian respects its own domain of validity provided the permissible values of $\bar{\rho}$ are chosen to be significantly smaller than unity when written in Planck units. In other words, the requirement $\sigma \ll 1$ essentially defines the domain of validity of the effective Hamiltonian. In contrast, in a purely classical geometrical description (standard inflationary scenario) such a restriction does not arise as the description itself a fundamental description of nature within the set-up of general relativity. Thus, it is clear from expression (41) that in this scenario the amplitude for the power spectrum of the density perturbation is naturally small. In other words, the small amplitude of the primordial density perturbation is a prediction of the framework of effective loop quantum cosmology.

It should be noted from equation (39) that if the equation of state is very close to -1 during the inflationary period then the amplitude of the density perturbation gets a large multiplicative factor at the time of horizon re-entry. In the case of loop quantum cosmology induced inflation, the equation of state (22) indeed is very close to -1 as $a \ll 1$ during its inflationary period. However in this scenario, one can still produce a small amplitude for the primordial density perturbation without fine tuning. One of the reasons behind this is the presence of the small factor $a^{4(1-n)}$ in the two-point density correlation function (34). The presence of this crucial small factor in the expression of the two-point density correlation function simply follows from the modified mode functions of the scalar field.

Another interesting property of the amplitude (41) is that it contains an exponential damping term $\mathrm{e}^{-\sigma}$. For the purpose of this paper the damping term is insignificant as $\sigma$ is required to be small in this paper. However, if one naively takes the energy scale to be of the order of the Planck scale even then the amplitude of the density perturbation will remain small as the exponential term becomes significant at that scale. In fact, this was the main motivation of the papers $[33,34]$.

Qualitatively, the smallness of the amplitude for the primordial density perturbation is readily predicted but to have a quantitative estimate one needs to choose some value for the associated energy scale. Assuming the density perturbation is adiabatic, i.e. it is the same as 
the curvature perturbation, one can relate the amplitude of the power spectrum of the density perturbation to the CMB angular power spectrum as follows [39]:

$$
\mathcal{A}^{2}=\left(\frac{3}{2}\right)^{2} \frac{9}{\left(2 \pi^{2}\right)} \frac{\tilde{l}(\tilde{l}+1) C_{\tilde{l}}^{\mathrm{AD}}}{2 \pi} .
$$

where $\tilde{l}$ is the multipole number of the angular power spectrum. We have also assumed that the relevant modes re-enter the horizon during the radiation-dominated era. The COBE data imply that $\frac{\tilde{l}(\tilde{l}+1) C_{l}^{\mathrm{AD}}}{2 \pi} \simeq 10^{-10}$. Using expression (41), one can easily deduce that $\sigma \approx 5.5 \times 10^{-3}$ (we have assumed here that at the end of loop quantum cosmology induced inflation, the radiation domination begins, i.e., $C_{\omega}=4$; the value of the ambiguity parameter $l$ is chosen to be $\frac{1}{2}$ ). It follows from the expression of $\sigma$ (24) that the corresponding energy density is $\bar{\rho} \approx\left(2.0 \times 10^{-3} M_{p}\right)^{4}=\left(2.0 \times 10^{16} \mathrm{GeV}\right)^{4}$. The associated energy scale comes down slightly if one assumes that the end of loop quantum cosmology induced inflation is followed by a standard accelerating phase (for example, if one takes $C_{\omega}=\frac{2}{3}\left(\omega=-\frac{1}{2}\right)$ then the corresponding energy density is $\left.\bar{\rho} \approx\left(0.8 \times 10^{-3} M_{p}\right)^{4}=\left(0.8 \times 10^{16} \mathrm{GeV}\right)^{4}\right)$.

The energy scale required to produce the observed amplitude of the density perturbation in effective loop quantum cosmology is not very different from the standard inflationary scenario where associated energy density is $\bar{\rho} \approx\left(2.0 \times 10^{16} \mathrm{GeV}\right)^{4}$ [39]. Then it is quite important to understand why is it necessary to fine tune field theoretical parameters in the standard scenario to produce a small amplitude. In this paper, we have considered a massive scalar field as a matter source. The energy density during the standard inflationary period is $\bar{\rho} \approx \frac{1}{2} m_{\phi}^{2} \phi^{2}$. In the standard inflationary scenario, to produce a sufficient amount of expansion (to solve the horizon problem and others), one needs to choose the values of the field to be $\phi^{2} \approx 10 M_{p}^{2}$ [39]. This in turn forces one to tune the mass parameter to $m_{\phi} \approx 10^{-6} M_{p}$ in order to produce a small amplitude for the primordial density perturbation. These fine tunings of field strength and the mass term are not only severe but also extremely difficult to sustain under standard quantum field theory. Specifically, sustaining such low mass parameters from loop corrections often requires new ingredients [47].

In other words, in the standard inflationary scenario, to get correct amplitude of density perturbation, the required fine tunings are directly related to the method by which inflation is realized, namely the imposition of slow-roll condition. One the other hand, in loop quantum cosmology the inflationary phase is realized generically [22] and not by imposing the slowroll condition. There is a physical understanding of it from the fundamental point of view. The famous singularity theorems tell us that in the cosmological set-up one cannot avoid an initial singularity if the matter contents always satisfy the strong energy condition (equation of state $\omega \geqslant-\frac{1}{3}$ ). Naturally, to avoid an initial singularity, the quantum gravity effects must drive the matter contents to effectively violate the strong energy condition, at least for a small enough volume. It has been shown that in loop quantum cosmology, one generically avoids a singularity at the quantum level [17] as well as the effective classical level [30-32]. Using the Raychaudhuri equation, it is then easy to see that the violation of strong energy conditions, i.e. $\omega<-\frac{1}{3}$ immediately implies an accelerating phase. In particular in [22], it has been proved that for any positive definite scalar potential, the loop quantum cosmology induced accelerating phase undergoes near exponential expansion. Thus, in loop quantum cosmology, the realization of an early accelerating phase is intimately related to the removal of the initial singularity. In this scenario, the inflation is essentially driven by the non-perturbative modification of the scalar field dynamics, namely via the spectrum of the inverse scale factor operator. This inflationary phase begins as soon as the system gets into the small volume regime (non-perturbative domain). Therefore, one is not required to fine tune the field strength 
sufficiently uphill as done in the standard scenario. Consequently, one is not required to fine tune field theoretical parameters to produce a small amplitude of density perturbation. Rather, as is shown in this paper, the small amplitude is a prediction of the framework that has been used in the calculation.

Nevertheless, one can impose a self-consistency requirement on the mass parameter in this calculation. Let us recall that in simplifying mode-function equation (28), we have neglected the mass term $\frac{m_{\phi}}{H}$ compared to the term $\frac{k}{H a}$. Since we are interested in calculating the power spectrum at the time of horizon crossing, i.e., $\frac{k}{\mathrm{Ha}}=2 \pi$, then to be self-consistent we must require that $m_{\phi}<2 \pi H$. This in turns implies that the maximum value of the mass parameter be $m_{\phi} \sim \frac{1}{27}(\bar{\rho})^{\frac{1}{4}}$. We may mention here that in effective loop quantum cosmology $\bar{\rho}$ in fact is the maximum energy density, i.e. $(\bar{\rho})^{\frac{1}{4}}$ is precisely the cut-off scale. It is worth emphasizing that the restriction on the mass parameter here is a self-consistency requirement of the calculation as solving the modified mode-function equation (28) including the mass term turns out to be not so easy a task.

\subsection{The spectral index}

So far in the calculation we have assumed that during the inflationary period energy density is strictly constant. But this was rather an approximation to simplify the calculation. We can in fact compute the effect of small variation of energy density. The small variation of the Hubble parameter leads to a small deviation from the scale-invariant power spectrum. The scale-dependent property of the power spectrum is conveniently described in terms of spectral index. From the conservation equation it follows that $\frac{\mathrm{d} \ln \rho}{\mathrm{d} \ln a}=-3\left(1+\omega^{\mathrm{eff}}\right)=$ $-C_{\omega}(n+2) a^{2(1-n)}$. Using this relation, one can compute the spectral index at the horizon crossing

$$
\begin{aligned}
n_{s}-1 & :=\frac{\mathrm{d} \ln \mathcal{P}_{\delta}(k)}{\mathrm{d} \ln k} \\
& \approx C_{\omega}(-n-2)\left(\frac{k}{2 \pi H}\right)^{2(1-n)}+4 c_{0}(1-n)\left(\frac{k}{2 \pi H}\right)^{4(1-n)} .
\end{aligned}
$$

We may note here that the spectral index $n_{s}$ is extremely close to unity and the difference $\left(n_{s}-1\right)$ depends non-trivially on the ambiguity parameter. But the most important property of the spectral index $(43)$ is $\left(n_{s}-1\right)>0$ for all allowed values of the ambiguity parameter $(0<l<1$, i.e., $-\infty<n<-2)$. This is in complete contrast to the standard single-field inflationary scenario. We have mentioned earlier that the power spectrum for both the scenarios varies as $H^{2}$. For the single-field standard inflationary scenario, the leading contribution to the spectral index deviation comes from the variation of Hubble parameter during the inflationary period. So for the standard scenario the spectral index is given by

$$
n_{s}-1:=\frac{\mathrm{d} \ln \mathcal{P}_{\delta}(k)}{\mathrm{d} \ln k} \approx-6 \epsilon
$$

where $\epsilon=\frac{1}{16 \pi G}\left(\frac{V^{\prime}(\phi)}{V(\phi)}\right)^{2}=4 \pi G \frac{\dot{\phi}^{2}}{H^{2}}$ is the slow-roll parameter of the standard inflationary scenario. In fact due to the time variation of $\epsilon$ there will be additional contributions in (44). But those will be sub-leading for the single-field inflationary scenario. Thus, for the single-field standard inflationary scenario the spectral index satisfies $\left(n_{s}-1\right)<0$.

It is worth pointing out that in the standard method (indirect method) of obtaining the power spectrum of the density perturbation, one first computes the power spectrum of the field fluctuation. Using expression (33), one can compute the power spectrum of the field 
fluctuation at the horizon exit, given by

$$
\mathcal{P}_{\phi}(k):=\frac{k^{3}}{2 \pi^{2}}\left|f_{k}\right|^{2}=\left(\frac{1}{2 \pi}\right) \frac{|n+2|}{3} \frac{n^{2} 2^{\frac{1}{n}}}{\Gamma\left(-\frac{1}{2 n}\right)^{2}} H^{2},
$$

which is scale invariant. In the standard inflationary scenario, this would have led to a scaleinvariant power spectrum of density perturbation. However, we have already mentioned that this is not straightforward in effective loop quantum cosmology. This is due to the fact that the question of how to obtain a general effective action, consequently an effective stress-energy tensor, from a canonical quantum theory of gravity is not yet well settled. This prevents one computing the power spectrum of density perturbation using standard method, as one needs to know the expression of the general effective stress-energy tensor, to relate the power spectrums of field fluctuation to the density perturbation. Nevertheless, one may naively assume that the power spectrum of the field fluctuation and that of the density perturbation will have a similar relation. In that case, the computed power spectrum of the density perturbation using the direct and indirect methods would differ from each other by an extremely weak $k$ dependence. This difference would then be similar in nature to the results of [33] in the context of the standard inflationary scenario. However, this difference would at most affect the quantitative nature (that too by a vanishingly small amount) but not the qualitative nature of the spectral index (43) whatsoever.

Thus, it is clear that for the effective loop quantum cosmology induced inflationary scenario the spectral index has a qualitatively distinct feature compared to that of the single-fielddriven standard inflationary scenario. In the following section, we discuss its observational consequences.

\subsection{Observational implications}

The power spectrum of the density perturbation generated during the inflationary era is not directly observable. Rather, the observed power spectrum corresponds to the density perturbation at the time of re-entry in the post-inflationary period. At the time of re-entry, the larger wavelength $\left(2 \pi k^{-1}\right)$ enters the horizon at a later time compared to the smaller wavelength. It is clear from expression (39) that if there is a change in the equation of state of the universe during re-entry then there will be an additional modification of the power spectrum. Since we are interested in estimating the original power spectrum generated during the inflationary era then its quite important to avoid additional modification of the power spectrum coming from other possible sources.

The observed anisotropy in the CMB sky corresponds to the density perturbation on the last scattering surface. The last scattering surface broadly demarcates the end of the radiationdominated era to the beginning of the matter-dominated era. Naturally, during this period $(1+\omega)$ changes from $\frac{4}{3}$ to 1 . While deriving expression (43) of the spectral index, we have assumed a constant equation of state during re-entry. Thus, for the purpose of comparison with observations, one must consider only those modes for which the equation of state was almost constant during re-entry. On the last scattering surface they will correspond to the modes which are well inside the horizon at the time of decoupling. Being smaller in wavelength these modes will subtend smaller angles in the present-day sky. Naturally these modes will correspond to the higher multipole number. Also if one considers sufficiently narrow bands in these parts of the spectrum then one can avoid additional modification coming from the sub-horizon evolution of the density perturbation in the period between their re-entry and the decoupling. 
To infer the property of the primordial density perturbation from the observed angular power spectrum of the $\mathrm{CMB}$, one needs to know the evolution of the universe for the period between the decoupling and the present-day universe. Since a major fraction of today's energy density is believed to come from the mysterious dark matter and dark energy then it is quite obvious that there will be a considerable influence from them on the inferred primordial power spectrum. The current observational estimate of the spectral index based on WMAP+SDSS data is $n_{s}=0.98 \pm 0.02$ [48-50]. This estimate is based on the entire part of the observed angular power spectrum; nevertheless, this agrees (rather marginally) with the expression for the spectral index (43) which is strictly valid only for the part of the spectrum in the higher multipole region. For this purpose, it may be more convenient to reconstruct the primordial power spectrum using the observed CMB angular power spectrum (for example, as done in [51-54]) and then consider the higher wavenumber part of the spectrum.

\section{Discussion}

In summary, we have computed the power spectrum of the density perturbation generated during the loop quantum cosmology induced inflationary phase. The resulting power spectrum is broadly scale invariant. Further, it has been shown that the small amplitude for primordial density perturbation is a natural prediction of the framework of effective loop quantum cosmology. Unlike the standard inflationary scenario, here one is not required to fine tune field theoretical parameters to produce the observed small amplitude for the density perturbation. The resulting power spectrum also has a qualitatively distinct feature compared to the standard single-field inflationary scenario. The spectral index in the effective loop quantum cosmology scenario satisfies $\left(n_{s}-1\right)>0$ whereas for the standard inflationary scenario it satisfies $\left(n_{s}-1\right)<0$.

Naturally, the spectral index of the power spectrum for the density perturbation generated during loop quantum cosmology induced inflation and the standard inflation differs in a nontrivial and non-overlapping way. This is a consequence of the fact that during loop quantum cosmology induced inflation the Hubble horizon shrinks marginally whereas in the standard inflationary scenario the Hubble horizon expands. This feature leads the power spectrum for the corresponding density perturbation to be tilted in opposite directions to each other. We have argued that this feature is a generic property of the corresponding scenario and not a property of some particular model. We have also pointed out the part of the observed CMB angular power spectrum that may be better suited for testing this particular feature observationally, namely the part corresponding to the higher multipole numbers of the CMB angular power spectrum.

It is worth pointing out that the calculation techniques used here within the adopted framework are analytic and the approximations used here are mostly justified. Nevertheless, one should keep it in mind that this calculation should not be considered as the first principle calculation of density perturbation within loop quantum cosmology. Rather, this calculation is based on effective loop quantum cosmology. Here, we have considered the non-perturbative modification of the kinetic term of the scalar matter Hamiltonian. In a first principle calculation (using an inhomogeneous model), one may naively expect to get corrections also in the gradient term of the matter Hamiltonian. This modification should depend on some ambiguity parameter similar to that of $l$ here. In this paper, it is shown that the ambiguity parameter $l$ dependence of the amplitude of the power spectrum is very weak. So the effect of such possible modifications on the amplitude of the power spectrum is expected to be rather small. The effect on spectral index deviation due to this modification is also expected to be small as it is determined mainly by the background evolution itself. Thus, it is very likely that the 
calculation presented here should be a good approximation of what is expected from a first principle calculation in the energy scale concerned.

We have used the direct method to compute the power spectrum of the density perturbation. This method uses the same techniques that are used in the standard quantum field theory. So one needs to have some kind of ultra-violet divergence regularization prescription. To regularize the ultra-violet divergence we have used the method outlined and explicitly shown in [33, 34]. This method relies on the assertion that a proper theory of quantum gravity should contain a zero-point proper length. In the effective loop quantum cosmology such a length scale is naturally available. In this method, regularization is essentially carried out by adding a zeropoint proper length to the standard definition of proper length. In this paper, the procedure was notationally simplified by using the notion of effective coordinate length. Nevertheless, the regularization procedure was carried out by hand. But this was expected as the calculations here were done using standard quantum field theory. On the other hand, one would expect that in a first principle calculation these regulators should come built-in as has been argued in the context of the full theory [35]. The crucial result of the paper, namely the properties of the spectral index are insensitive to the regularization method as it is dominantly determined by the nature of the effective equation of state during its inflationary phase.

In the standard inflationary scenario one is also interested in computing the power spectrum for the tensor mode of the metric fluctuations, i.e., a gravitational wave. But, as we have mentioned, the technology required to deal with inhomogeneity at the fundamental level in loop quantum cosmology is not available yet. In the loop quantum gravity approach, geometry is quantized in non-perturbative way. Thus, it is not very easy to 'guess' the structure of the quantum fluctuation of geometry until one does an explicit calculation within the framework. Nevertheless, one would naively expect that the power spectrum for the tensor mode perturbation should be similar to the standard scenario as the structure of the effective gravity sector Hamiltonian is similar to the classical Hamiltonian in the relevant length scale, apart from the fact that the relevant energy scale during corresponding inflationary period is also similar.

Before we discuss the implications of possible outcomes of the mentioned test, let us compare the standard inflationary scenario and loop quantum cosmology induced inflationary scenario. In order to have a successful inflation in the standard scenario, generally one requires multi-level fine tuning of the field parameters. In other words, one faces several kinds of naturalness problems to achieve a successful inflation.

The first is to start inflation. In the standard inflationary scenario, it is necessary to choose initial field velocity to be sufficiently small so that the equation of state $\frac{\frac{1}{2} \dot{\phi}^{2}-V(\phi)}{\frac{1}{2} \dot{\phi}^{2}+V(\phi)} \approx-1$. The second is to sustain inflation. In the standard scenario, one is required to choose the field potential to be flat enough so that field does not gain momentum quickly. The third is to generate sufficient expansion (to solve the horizon problem and others). To achieve this in the standard scenario, one is required to choose the initial field configuration sufficiently uphill in the potential. In other words, one is required to fine tune the initial field configuration. The fourth is to end inflation. In many cases, this requires a sort of potential engineering to have a long flat plateau and then a fast fall-off in the potential profile. The fifth is to produce small amplitude for primordial density perturbation. To produce the observed small amplitude of density perturbation one needs to fine tune parameters of the field potential. This fine tuning is basically required to compensate the 'third' fine tuning.

On the other hand, to achieve the first, second and fourth requirements in the loop quantum cosmology induced inflationary scenario one does not require the parameters to be fine tuned. These requirements are naturally achieved as they simply follow from the spectrum of the 
inverse scale factor operator. The fifth requirement, i.e. the small amplitude, as shown in this paper, is a natural prediction of the effective loop quantum cosmology. The situation regarding the third problem also improves significantly. In loop quantum cosmology, the generated amount of expansion is controlled by the ambiguity parameter $j$. Clearly, to produce sufficiently large expansion, using loop quantum cosmology alone, one is required to choose the value of $j$ to be large. Thus, it is very likely that only the initial part of the inflation was driven by loop quantum cosmology modification. It has been argued in [40-42] that the loop quantum cosmology induced inflationary phase can lead to a secondary standard inflationary phase. This follows from the fact that the in-built inflationary period of loop quantum cosmology can produce favourable initial conditions for an additional standard inflationary phase. In [41], the authors have also studied the possible effects of the above mechanism on the CMB angular power spectrum generated during the standard inflationary phase that follows the LQC-induced inflationary phase and shown that it can lead to suppression of power in the low CMB multipoles. Since the observed part of the CMB angular power spectrum generally corresponds to the early period of inflation then it may well be the situation where the observed part of the CMB angular power spectrum corresponds to the loop quantum cosmology driven inflationary period.

It is worthwhile emphasizing that the large amount of expansion in this scenario is required not to solve the horizon problem (being non-singular this model avoids horizon problem [22]) rather to avoid a different kind of problem. We have seen that the 'initial size' of the universe was typically of the order of Planck units and the corresponding energy scale was also typically of the order of Planck units. During the relativistic particle (radiation) dominated era energy scale falls off typically with the inverse power of the associated length scale. It is then difficult to understand why the universe is so large $\left(\sim 10^{60} L_{p}\right)$ today but still has relatively a very high energy scale $\left(\sim 10^{-30} M_{p}\right)$. During the inflationary period, on the other hand, the energy scale remains almost constant whereas the length scale grows almost exponentially with coordinate time. It is now clear that we can avoid this discrepancy between the energy scale and length scale of the universe provided there exists an inflationary period with sufficiently long duration in the early universe.

Now if the observed power spectrum turns out to be not in agreement with the computed power spectrum, then one should conclude that the phase of inflation corresponding to the observed window could not possibly be driven by loop quantum cosmology modification. It may then restrict the allowed choices for the ambiguity parameter $j$. Consequently, it will be an important issue to understand within the framework of isotropic loop quantum cosmology with minimally coupled scalar matter field, why the observed universe today is so large but still has sufficiently high energy scale.

\section{Acknowledgments}

I thank Ghanashyam Date and Martin Bojowald for helpful discussions and critical comments on the manuscript. I thank Martin Bojowald for a cordial invitation to visit Max-PlanckInstitut für Gravitationsphysik where a major part of this work was completed. I also thank the anonymous referee whose constructive as well as instructive criticisms led to a significant improvement in the paper and illumination of several conceptual issues.

\section{References}

[1] Bardeen J M, Steinhardt P J and Turner M S 1983 Spontaneous creation of almost scale-free density perturbations in an inflationary universe Phys. Rev. D 28679 
[2] Brandenberger R H 2000 Inflationary cosmology: progress and problems Invited lectures at International School on Cosmology (Kish Island, Iran, 22 January 22-4 February 1999) (Dordrecht: Kluwer) (Preprint hep-ph/9910410) to be published

[3] Narlikar J V and Padmanabhan T 1991 Ann. Rev. Astron. Astrophys. 29325

[4] Adams F C, Freese K and Guth A H 1991 Constraints on the scalar field potential in inflationary models Phys. Rev. D 43965

[5] Bojowald M 2000 Loop quantum cosmology: I. Kinematics Class. Quantum Grav. 17 1489 (Preprint gr-qc/9910103)

[6] Bojowald M 2000 Loop quantum cosmology: II. Volume operators Class. Quantum Grav. 171509 (Preprint gr-qc/9910104)

[7] Bojowald M 2001 Loop quantum cosmology: III. Wheeler-De Witt operators Class. Quantum Grav. 181055 (Preprint gr-qc/0008052)

[8] Bojowald M 2001 Loop quantum cosmology: IV. Discrete time evolution Class. Quantum Grav. 181071 (Preprint gr-qc/0008053)

[9] Bojowald M 2002 Isotropic loop quantum cosmology Class. Quantum Grav. 192717 (Preprint gr-qc/0202077)

[10] Bojowald M 2001 The semiclassical limit of loop quantum cosmology Class. Quantum Grav. 18 L109 (Preprint gr-qc/0105113)

[11] Bojowald M and Morales-Tecotl H A 2004 Cosmological applications of loop quantum gravity (Lecture Notes in Physics vol 646) p 421 (Preprint gr-qc/0306008)

[12] Ashtekar A, Bojowald M and Lewandowski J 2003 Mathematical structure of loop quantum cosmology Adv. Theor. Math. Phys. 7233 (Preprint gr-qc/0304074)

[13] Rovelli C 1998 Loop quantum gravity Living Rev. Rel. 11 (Preprint gr-qc/9710008)

[14] Thiemann T 2001 Introduction to modern canonical quantum general relativity Preprint gr-qc/0110034

[15] Thiemann T 2003 Lectures on loop quantum gravity (Lecture Notes in Physics vol 631) p 41 (Preprint gr-qc/0210094)

[16] Ashtekar A and Lewandowski J 2004 Background independent quantum gravity: a status report Class. Quantum Grav. 21 R53 (Preprint gr-qc/0404018)

[17] Bojowald M 2001 Absence of singularity in loop quantum cosmology Phys. Rev. Lett. 865227 (Preprint gr-qc/0102069)

[18] Bojowald M 2003 Homogeneous loop quantum cosmology Class. Quantum Grav. 202595 (Preprint gr-qc/0303073)

[19] Bojowald M and Date G 2004 Quantum suppression of the generic chaotic behavior close to cosmological singularities Phys. Rev. Lett. 92071302 (Preprint gr-qc/0311003)

[20] Bojowald M, Date G and Hossain G M 2004 The Bianchi IX model in loop quantum cosmology Class. Quantum Grav. 213541 (Preprint gr-qc/0404039)

[21] Bojowald M 2002 Inflation from quantum geometry Phys. Rev. Lett. 89261301 (Preprint gr-qc/0206054)

[22] Date G and Hossain G M 2005 Genericness of inflation in isotropic loop quantum cosmology Phys. Rev. Lett. 94011301 (Preprint gr-qc/0407069)

[23] Bojowald M 2001 The inverse scale factor in isotropic quantum geometry Phys. Rev. D 64084018 (Preprint gr-qc/0105067)

[24] Bojowald M 2002 Quantization ambiguities in isotropic quantum geometry Class. Quantum Grav. 195113 (Preprint gr-qc/0206053)

[25] Birrell N D and Davies P C W 1982 Quantum Fields in Curved Space (Cambridge: Cambridge University Press)

[26] Ford L H 1997 Quantum field theory in curved spacetime Preprint gr-qc/9707062

[27] Hossain G M 2004 Hubble operator in isotropic loop quantum cosmology Class. Quantum Grav. 21 179 (Preprint gr-qc/0308014)

[28] Bojowald M, Singh P and Skirzewski A 2004 Time dependence in quantum gravity Preprint gr-qc/0408094

[29] Date G and Hossain G M 2004 Effective Hamiltonian for isotropic loop quantum cosmology Class. Quantum Grav. 214941 (Preprint gr-qc/0407073)

[30] Singh P and Toporensky A 2004 Big crunch avoidance in $k=1$ loop quantum cosmology Phys. Rev. D 69 104008 (Preprint gr-qc/0312110)

[31] Vereshchagin G V 2004 Qualitative approach to semi-classical loop quantum cosmology J. Cosmol. Astropart. Phys. JCAP07(2004)013 (Preprint gr-qc/0406108)

[32] Date G and Hossain G M 2005 Genericness of big bounce in isotropic loop quantum cosmology Phys. Rev. Lett. 94011302 (Preprint gr-qc/0407074)

[33] Padmanabhan T, Seshadri T R and Singh T P 1989 Making inflation work: damping of density perturbations due to Planck energy cutoff Phys. Rev. D 392100 
[34] Padmanabhan T 1988 Acceptable density perturbations from inflation due to quantum gravitational damping Phys. Rev. Lett. 602229

[35] Thiemann T 1998 QSD V: quantum gravity as the natural regulator of matter quantum field theories Class. Quantum Grav. 151281 (Preprint gr-qc/9705019)

[36] Bojowald M 2004 Loop quantum cosmology: recent progress Pramana 63765 (Preprint gr-qc/0402053)

[37] Bowman F 1958 Introduction to Bessel Functions (New York: Dover) p 117

[38] Bardeen J M 1980 Gauge invariant cosmological perturbations Phys. Rev. D 221882

[39] Riotto A 2002 Inflation and the theory of cosmological perturbations Lectures given at ICTP Summer School on Astroparticle Physics and Cosmology (Trieste, Italy, 17 June-5 July 2002) (Preprint hep-ph/0210162) (Published in 2002 Trieste Astropart. Phys. Cosmol. 317-413)

[40] Bojowald M and Vandersloot K 2003 Loop quantum cosmology, boundary proposals, and inflation Phys. Rev. D 67124023 (Preprint gr-qc/0303072)

[41] Tsujikawa S, Singh P and Maartens R 2004 Loop quantum gravity effects on inflation and the CMB Class. Quantum Grav. 215767 (Preprint astro-ph/0311015)

[42] Bojowald M, Lidsey J E, Mulryne D J, Singh P and Tavakol R 2004 Inflationary cosmology and quantization ambiguities in semi-classical loop quantum gravity Phys. Rev. D 70043530 (Preprint gr-qc/0403106)

[43] Martin J and Brandenberger R H 2001 The trans-Planckian problem of inflationary cosmology Phys. Rev. D 63 123501 (Preprint hep-th/0005209)

[44] Niemeyer J C 2001 Inflation with a high frequency cutoff Phys. Rev. D 63123502 (Preprint astro-ph/0005533)

[45] Tsujikawa S, Maartens R and Brandenberger R 2003 Non-commutative inflation and the CMB Phys. Lett. B 574141 (Preprint astro-ph/0308169)

[46] Brandenberger R and Ho P M 2002 Noncommutative spacetime principle stringy spacetime uncertainty and fluctuations density Phys. Rev. D 66023517 (2002 AAPPS Bull. 12N1 10) (Preprint hep-th/0203119)

[47] Liddle A R and Lyth D H 2000 Cosmological Inflation and Large-Scale Structure (Cambridge: Cambridge University Press)

[48] Tegmark M 2004 What does inflation really predict? (Preprint astro-ph/0410281)

[49] Tegmark M et al (SDSS Collaboration) 2004 Cosmological parameters from SDSS and WMAP Phys. Rev. D 69103501 (Preprint astro-ph/0310723)

[50] Seljak U et al 2004 Cosmological parameter analysis including SDSS Ly-alpha forest and galaxy bias: constraints on the primordial spectrum of fluctuations, neutrino mass, and dark energy Preprint astro-ph/ 0407372

[51] Bridle S L, Lewis A M, Weller J and Efstathiou G 2003 Reconstructing the primordial power spectrum Mon. Not. R. Astron. Soc. 342 L72 (Preprint astro-ph/0302306)

[52] Mukherjee P and Wang Y 2003 Model-independent reconstruction of the primordial power spectrum from WMAP data Astrophys. J. 5991 (Preprint astro-ph/0303211)

[53] Hannestad S 2004 Reconstructing the primordial power spectrum - a new algorithm J. Cosmol. Astropart. Phys. JCAP04(2004)002 (Preprint astro-ph/0311491)

[54] Shafieloo A and Souradeep T 2004 Primordial power spectrum from WMAP Phys. Rev. D 70043523 (Preprint astro-ph/0312174) 